Identifying QTL and genetic correlations between fur quality traits in mink (Neovison vison)

Thirstrup, Janne Pia; Anistoroaei, Razvan Marian; Guldbrandtsen, Bernt; Christensen, Knud Arnbjerg; Fredholm, Merete; Nielsen, V. H.

Published in:

Animal Genetics

DOI:

10.1111/age.12102

Publication date:

2014

Document version

Early version, also known as pre-print

Citation for published version (APA):

Thirstrup, J. P., Anistoroaei, R. M., Guldbrandtsen, B., Christensen, K. A., Fredholm, M., \& Nielsen, V. H. (2014). Identifying QTL and genetic correlations between fur quality traits in mink (Neovison vison). Animal Genetics, 45(1), 105-110. https://doi.org/10.1111/age.12102 


\title{
Identifying QTL and genetic correlations between fur quality traits in mink (Neovison vison)
}

\author{
J. P. Thirstrup*, R. Anistoroaei ${ }^{\dagger}$, B. Guldbrandtsen*, K. Christensen ${ }^{\dagger}$, M. Fredholm ${ }^{\dagger}$ and \\ V. H. Nielsen* \\ *Department of Molecular Biology and Genetics, Faculty of Science and Technology, Aarhus University, Tjele, Denmark. 'Department of \\ Basic Animal and Veterinary Sciences, Faculty of Science, University of Copenhagen, Copenhagen, Denmark.
}

\section{Summary}

\section{Introduction}

Fur quality is very important in mink production as, together with the size, it determines the price of the skin. Fur quality is a composite trait, including color and purity, structure and length of guard hair and density of the wool. The characteristics that cause a fur to be classified as high quality are difficult to quantify. One skin might be classified as high quality due to a silky appearance of the fur, and another due to a high density of the wool. It is therefore of interest to understand which single traits influence the classification as high quality. Besides the construction of genomic resources, that is, a genetic map (Anistoroaei et al. 2009) and BAC library (Anistoroaei et al. 2011) in mink,

Address for correspondence

J. P. Thirstrup, Department of Molecular Biology and Genetics, Faculty of Science and Technology, Aarhus University, Tjele, Denmark.

E-mail: janne.thirstrup@agrsci.dk

Accepted for publication 10 February 2013 most genomic work so far has focused on identifying coat color genes (Anistoroaei \& Christensen 2007; Anistoroaei et al. 2008, 2012a,b; Benkel et al. 2009). In this study, we have focused on traits of economic value such as guard hair length, guard hair thickness, density of wool and appearance of the surface of the fur as well as the size of the skin.

The aims of this study were to (i) detect QTL affecting fur quality traits in mink and their mode of gene action and (ii) estimate phenotypic and genotypic correlations in order to analyze whether traits with similar QTL positions share similar genetic background.

\section{Materials and methods}

\section{Animal material}

A reciprocal $\mathrm{F}_{2}$ design crossing Nordic Brown mink and American Black short nap mink was used. The two lines were selected based on their distinct phenotypes (Table 1) and are expected to be genetically different as well. Mink from the Nordic Brown line were large with coarse and long 
Table 1 Least-square mean of phenotypic values for males in the two parental lines, the $F_{1}$ generation and the $F_{2}$ generation. Effects of the females averaged across generations are given. Standard errors are given in brackets.

\begin{tabular}{lrrrr}
\hline & Nordic Brown type & Short nap & Effect of sex & $F_{1}$ \\
\hline Guard hair thickness & $3.80(0.16)$ & $0.82(0.16)$ & $0.24(0.05)$ & $2.66(0.06)$ \\
Guard hair length & $5.92(0.27)$ & $1.21(0.26)$ & $0.27(0.07)$ & $3.96(0.10)$ \\
Wool density & $4.01(0.21)$ & $2.27(0.21)$ & $0.23(0.06)$ & $2.94(0.08)$ \\
Surface & $4.72(0.24)$ & $2.45(0.24)$ & $-0.12(0.07)$ & $3.48(0.06)$ \\
Quality & $9.91(0.40)$ & $4.78(0.40)$ & $-0.43(0.11)$ & $3.07(0.05)$ \\
Skin length & $92.07(0.78)$ & $84.88(0.78)$ & $-17.21(0.23)$ & $3.44(0.05)$ \\
\hline
\end{tabular}

guard hair. They were brown with reddish wool of poor quality. The line has been selected for large size for several generations (Nielsen et al. 2011) at Research Centre Foulum in Denmark. The American short nap mink were small with silky and short guard hair and dense wool of good quality. The short nap mink were black, with short heads and blunt snouts. The line was imported to Denmark for breeding from Patrick X, Nova Scotia, Canada. Mink from this line were bought for the QTL study performed at Research Centre Foulum.

Five males from each line, 16 females from the Nordic Brown line and 20 females from the American Black line, were used for the parental generation. To establish the $F_{1}$ generation, the two lines were crossed reciprocally: Short nap males were mated to Nordic Brown females (block A), and Nordic Brown males were mated to short nap females (block B). Each male was mated to a maximum of five females. To increase litter size, 2-year-old Brown females were used in the parental generation. Relationship between parents was minimized: Mothers of the males were avoided and none of the female mink were sibs. The $\mathrm{F}_{2}$ generation was established by mating both within and between blocks. These matings were performed using 31 males and 108 females. To ensure sufficient power for statistical tests (Weller et al. 1990), the same mink from the $\mathrm{F}_{1}$ generation were mated in two succeeding years, providing 933 mink in the $\mathrm{F}_{2}$ generation.

\section{Genotyping}

In total, 1082 mink (parental, $\mathrm{F}_{1}$ and $\mathrm{F}_{2}$ ) were genotyped. Spleens were sampled at pelting from all breeding animals in the parental and the $\mathrm{F}_{1}$ generation and from all animals in the $\mathrm{F}_{2}$ generation. DNA was extracted using a salting-out protocol (modified from Miller et al. 1988). One hundred and four microsatellite markers positioned with an average distance of approximately $11 \mathrm{cM}$, as described by Anistoroaei et al. (2012c), were used for the study. Genotyping was performed as previously described in Anistoroaei et al. (2012c).

\section{Genetic linkage map}

Data from this study were used to improve the first published mink linkage map (Anistoroaei et al. 2009) by also adding more microsatellite markers (Anistoroaei et al. 2012c).

\section{Phenotyping}

Phenotypes were recorded for all genotyped animals (1082 mink). Recordings were performed at Kopenhagen Fur by professional skin sorters. All skins prepared for this analysis were graded according to the standard procedures. All mink from the three generations were phenotyped simultaneously. Each trait was assessed within the same day by the same skin sorter. Due to diverging expression of quality, males and females were separated before phenotyping.

Twelve phenotypic traits were recorded according to the standard procedures. Some of the traits were connected to coloration, but in our study we focused on genes influencing the structure of the fur. In this study, results for guard hair thickness, guard hair length, wool density, surface of the fur, fur quality and skin length are presented. Guard hair thickness was measured in four categories from finest (1) to thickest (4). Guard hair length was recorded as the length of hair protruding from the wool. It was measured in seven categories from very short (1) to very long (7). The wool (underfur) density was evaluated in six categories from the highest density (1) to the lowest density of wool (6). Fur surface was measured in seven categories from the silkiest (1) to the most coarse with thick guard hair (7). Recording of fur quality was a subjective composite depending on many characteristics, including color, hair length, hair thickness and wool density. It was evaluated in 12 categories from best (1) to poorest (12). Skin length was measured in intervals of $6 \mathrm{~cm}$. Visual inspection of histograms did not indicate any strong deviation from normality. We therefore assumed that the underlying distributions of the phenotypes were normal.

For the calculation of generation phenotypic least-square mean and estimation of genetic and phenotypic correlation as well as heritability, 1268 animals were included (thus males from the $\mathrm{F}_{1}$ generation not used for breeding and females without any offspring were included in this material).

\section{Data analysis}

Least-square means of phenotypic values were estimated for the two parental populations and the $\mathrm{F}_{1}$ and $\mathrm{F}_{2}$ generations. A linear model including the effect of sex and populations was used for estimation of the means. 


$$
Y_{i j}=\mu+\operatorname{sex}_{i}+\operatorname{pop}_{j}+e_{i j},
$$

where $\mathrm{Y}_{i j}$ is phenotypic mean of the trait for the ith sex and the $j$ th generation, $\operatorname{sex}_{i}$ is fixed effect of gender $(i=$ males and females), pop $_{j}$ is fixed effect of population $(j=$ Nordic Brown mink, American short nap, $\mathrm{F}_{1}$ and $\mathrm{F}_{2}$ ), and $\mathrm{e}_{i j}$ is random error. The parental population consisted of the two mink color types, and the $\mathrm{F}_{2}$ generation born in 2006 and 2007 was pooled into one population. The $\mathrm{R}$ package, function $\mathrm{lm}$, version 4.5-3 (Ihaka \& Gentleman 1996), was used for the estimations. Significance of effect of sex and population was inferred from value \pm 1.96 S.E. which did not include zero

Levels of inbreeding $(\mathrm{F})$ within and genetic distance $\left(\mathrm{F}_{\mathrm{ST}}\right)$ between the two lines were estimated from the genetic markers using Genalex 6.41 (Peakall \& Smouse 2006).

The QTL analyses were performed by a least-square regression method developed by Haley et al. (1994) for crosses between outbreed lines and implemented in the software program GRIDQTL (Seaton et al. 2002). The option of fitting a single QTL was used. A model including the effects of sex and year was used. Categorical classifications as described above were used as response variables. A chromosome-wise significance level of 0.01 , corresponding to a LOD score threshold of 3.7, was used (Weller et al. 1990). Permutation tests with 1000 permutations were used for estimating the significance level (Churchill \& Dorge 1994). Confidence intervals were estimated using bootstrap analyses with 10000 iterations (Visscher et al. 1996).

Additive and dominant coefficients for the QTL were estimated as explained in Haley et al. (1994). Assuming the two lines were fixed for alternative alleles, the additive effect is half the difference between the two homozygote lines and the dominance effect is the deviation from the mean of the two homozygotes. A positive additive effect indicates that alleles from the parental Nordic Brown mink increase the trait value on the categorical scale. Modes of gene action were assessed from additive and dominance effects according to Falconer \& Mackay (1996). The values of the dominant effect relative to the additive effect indicated whether the alleles act in an additive or dominant fashion.

To analyze whether traits related to hair structure with similar QTL positions share some of their genetic background, we estimated the genetic and phenotypic correlations between the traits. Estimates of genotypic and phenotypic correlations as well as heritabilities were performed in bivariate models using the average information-restricted maximum-likelihood (AI-REML) algorithm implemented in the DMU package (Madsen \& Jensen 2010). The following model was used for all traits:

$$
Y_{i j}=\operatorname{Sex}_{i}+\operatorname{Year}_{j}+\operatorname{Sex}_{i} \times \operatorname{Year}_{j}+\operatorname{Animal}_{i j}+\mathrm{e}_{i j},
$$

where $Y_{i j}$ is the phenotypic record for the ith sex and the $j$ th production year, Sex is the fixed effect of gender $(i=$ male or female), Year is the fixed effect of production year $(j=2004 \ldots .2007)$, Animal $_{i j}$ is the random additive genetic animal effect, and $\mathrm{e}_{i j}$ is random residual. Assumptions for random effects are given below:

$$
\left[\begin{array}{c}
\text { Animal } \\
\mathrm{e}
\end{array}\right] \sim\left(\left[\begin{array}{l}
0 \\
0
\end{array}\right],\left[\begin{array}{cc}
\mathrm{G}_{0} \otimes \mathrm{A} & 0 \\
0 & \mathrm{R}_{0} \otimes \mathrm{I}
\end{array}\right]\right),
$$

where $G_{0}$ and $R_{0}$ are the additive genetic and the residual covariance matrices respectively for the traits in the actual analysis and A is the additive relationship matrix setup assuming animals in the base population were unrelated.

Asymptotic standard errors of estimated (co)variance components were obtained from the average information matrix. For quantities derived from covariance components (heritabilities and correlations), standard errors were obtained using a Taylor series approximation.

\section{Results}

Least-square means of phenotypic values are given in Table 1. Values for males from the two parental lines, for the $F_{1}$ and $F_{2}$ generation, as well as the effect of the female sex averaged across three generations are given with standard errors (SE). Short nap mink had lower phenotypic values compared to Nordic Brown mink. The effects of sex and generations were significantly different from zero.

The lines were not inbred: $\mathrm{F}_{\text {Nordic Brown mink }}$ (SE) was $-0.014(0.023)$, and $\mathrm{F}_{\text {American short nap mink }}(\mathrm{SE})$ was 0.040 (0.023). $\mathrm{F}_{\mathrm{ST}}$ between Nordic Brown mink and American short nap mink was 0.071 , indicating that the lines were genetically differentiated.

We found evidence for QTL affecting guard hair thickness on five chromosomes, guard hair length on three chromosomes, wool density on two chromosomes, surface on three chromosomes, quality on four chromosomes and skin length on two chromosomes. Thus, QTL affecting the traits were found on nine of the 14 mink autosomes. Positive additive effects for most traits indicate that alleles originating from the Brown mink line provide longer and thicker guard hair, lower density of wool, poorer surface and lower quality.

QTL affecting guard hair thickness were found on chromosomes 1, 2, 3, 6 and 13 (Table 2). On chromosomes 1, 2 and 3, alleles acted additively. On chromosome 6, the allele from the Nordic Brown mink acted dominantly to the short nap allele, and on chromosome 13, the alleles acted in an overdominant fashion. The sizes of additive and dominance effects are given in Table 2 .

On chromosomes 2, 3 and 6, QTL affecting guard hair length were detected. On chromosomes 2 and 3, alleles acted additively. On chromosome 6, the alleles originating from the Brown mink were dominant to the short nap alleles.

QTL affecting wool density were found on chromosomes 6 and 13. On chromosome 6 , the alleles acted in an 
Table 2 Chromosome number, chromosomal position with 95\% confidence intervals (CI), LOD score, additive and dominance effect with standard errors (SE) for guard hair thickness, guard hair length, wool density surface, quality and skin length.

\begin{tabular}{lcclrr}
\hline & Chromosome number & Location CM $(95 \% \mathrm{Cl})$ & Lod-score & Additive effect (SE) & Dominance effect (SE) \\
\hline Guard hair thickness & 1 & $0(0.0-100.0)$ & 3.85 & $0.22(0.05)$ & $0.0(0.09)$ \\
& $2^{\mathrm{A}}$ & $58(10.0-120.0)$ & 6.48 & $0.20(0.05)$ & $-0.06(0.08)$ \\
& $3^{\mathrm{B}}$ & $9(2.0-79.0)$ & 7.29 & $0.30(0.05)$ & $-0.03(0.08)$ \\
Guard hair length & $6^{\mathrm{C}}$ & $36(19.0-53.0)$ & 6.55 & $0.27(0.06)$ & $0.21(0.08)$ \\
& 13 & $27(0.0-63.0)$ & 3.7 & $0.14(0.08)$ & $0.33(0.09)$ \\
Wool density & $2^{\mathrm{A}}$ & $58(11.0-60.0)$ & 9.26 & $0.31(0.05)$ & $-0.11(0.09)$ \\
Surface & $3^{\mathrm{B}}$ & $9(0.0-79.0)$ & 4.23 & $0.22(0.06)$ & $-0.14(0.09)$ \\
& $6^{\mathrm{C}}$ & $36(19.0-53.0)$ & 7.38 & $0.24(0.06)$ & $0.27(0.08)$ \\
Quality & $6^{\mathrm{D}}$ & $23(11.0-40.0)$ & 5.34 & $0.05(0.06)$ & $0.31(0.08)$ \\
& $13^{\mathrm{E}}$ & $83(0.0-83.0)$ & 3.97 & $0.28(0.09)$ & $0.27(0.12)$ \\
& $7^{\mathrm{F}}$ & $34(33.0-63.5)$ & 3.7 & $0.44(0.11)$ & $0.14(0.14)$ \\
& 12 & $23(7.0-55.0)$ & 5.9 & $-0.37(0.08)$ & $0.23(0.13)$ \\
Skin length & 13 & $10(0.0-83.0)$ & 5.81 & $-0.38(0.13)$ & $0.04(0.15)$ \\
& $6^{\mathrm{D}}$ & $25(20.0-53.0)$ & 5.19 & $0.41(0.12)$ & $0.50(0.15)$ \\
& $7^{\mathrm{F}}$ & $39(34.0-49.0)$ & 6.47 & $0.85(0.19)$ & $0.79(0.25)$ \\
& 11 & $75(5.0-108.0)$ & 4.4 & $0.36(0.12)$ & $0.20(0.17)$ \\
\end{tabular}

A-F: Traits with same letter share the same QTL position.

overdominant way. On chromosome 13, alleles from the Brown mink were dominant to short nap alleles.

QTL affecting the fur surface were found on chromosomes 7, 12 and 13. On chromosomes 7 and 13, alleles acted additively. Alleles on chromosomes 12 and 13 originating from the Brown mink decreased the phenotypic value. On chromosome 12, alleles originating from short nap were dominant to alleles originating from Brown mink.

QTL affecting fur quality were found on chromosomes 6, 7, 11 and 13. All alleles originating from the Brown mink were dominant to alleles originating from the short nap mink.

QTL affecting skin length were found on chromosomes 7 and 9. On chromosome 7, alleles acted additively. On chromosome 9, the alleles originating from short nap were dominant to alleles originating from Brown mink.

Some QTL for different traits were located either at the same position or in close proximity to each other (Table 2). QTL for both guard hair thickness and guard hair length were located on chromosome 2 (58 cM, Mvi1843), on chromosome 3 (9 cM, Mvi1007) and on chromosome 6 (36 cM, Mvi4052). For wool and quality, QTL were detected on chromosome 6 (23 and $25 \mathrm{cM}$, Mvi4063 and Mvi192 respectively) and on chromosome 13 (83 cM, RAN26). For surface, quality and skin length, QTL were found on chromosome 7 (34, 39 and $37 \mathrm{cM}$, Mvi1957). However, all positions were estimated with rather wide confidence intervals. Given that traits sharing the same QTL position might also be genetically correlated, analyses were performed to support the findings of shared QTL positions. Correlations between the traits are given in Table 3. The genetic correlation between guard hair length and guard hair thickness was significant and high. Quality was significantly correlated with guard hair thickness, density of wool and surface. Guard hair thickness and wool were significantly correlated as well. The phenotypic correlations were of the same orders of magnitude as the genetic correlations. The heritabilities $\left(\mathrm{h}^{2}\right)$ were significantly different from zero, ranging from $0.15(0.04)$ to $0.43(0.07)$.

\section{Discussion}

The QTL analyses performed in this study detected chromosomal areas harboring genes affecting fur quality traits with emphasis on hair structure and skin length. We also revealed the mode of gene action and strong evidence for correlation between the traits examined and between single traits and quality. The results of our analyses indicate that either pleiotropy or gametic phase disequilibrium between alleles is of importance for the inheritance of some of the traits.

We found QTL affecting guard hair length, guard hair thickness, wool density, surface, quality and skin length on nine of the 14 mink autosomes. In total, we found 19 QTL affecting fur quality traits at a chromosome-wise significance level of $P \leq 0.01$. The signs of the additive effects were consistent with the direction of phenotypic differences between the parental lines. Alleles originating from the Nordic Brown mink provide longer and thicker guard hair, lower density of wool, a poorer surface, lower quality and an increased skin length compared to the short nap mink. We found that QTL affecting some of the traits were located either at the same position or in close proximity to each other. Guard hair length and guard hair thickness, wool 
Table 3 Estimates of genetic and phenotypic correlations and heritabilities for guard hair thickness, guard hair length, quality, surface and wool density. Genetic correlations are shown above the diagonal, phenotypic correlation below the diagonal and heritabilities in the diagonal. Standard errors (SE) are given in brackets. Significant correlations are shown in boldface.

\begin{tabular}{|c|c|c|c|c|c|}
\hline & Guard hair thickness & Guard hair length & Wool density & Surface & Quality \\
\hline Guard hair thickness & $0.411(0.065)$ & $0.880(0.080)$ & $0.424(0.117)$ & $0.205(0.139)$ & $0.468(0.111)$ \\
\hline Guard hair length & 0.423 & $0.153(0.043)$ & $0.301(0.166)$ & $0.100(0.180)$ & $0.121(0.177)$ \\
\hline Wool density & 0.259 & 0.177 & $0.430(0.068)$ & $0.150(0.144)$ & $0.742(0.075)$ \\
\hline Surface & 0.152 & 0.009 & 0.170 & $0.326(0.059)$ & $0.326(0.059)$ \\
\hline Quality & 0.344 & 0.107 & 0.586 & 0.336 & $0.337(0.053)$ \\
\hline
\end{tabular}

density and quality as well as surface and quality share similar QTL positions. Guard hair thickness and guard hair length also are highly genetically correlated, as are wool density and quality as well as wool density and guard hair thickness. This might indicate that these traits to some extent share a common genetic background.

Quality is a composite trait and reflects the combination of different single traits. This study suggests that quality is phenotypically and genetically correlated with wool density, surface and guard hair thickness, consistent with these traits being important for a good fur quality. Fur of high quality is fur for which the guard hair lies in the same direction, not protruding in all directions. Dense wool is able to support the guard hair so that the appearance of the fur appears harmonious. In the study of Kenttämies \& Vilva (1988), the phenotypic correlation between underfur density and general appearance was high, consistent with our findings. Some of the QTL affecting quality are in the same positions as are QTL affecting wool density, surface and guard hair thickness, indicating that the traits may be under the influence of some of the same genes or of genes positioned in close physical proximity. Also, wool density and guard hair thickness are phenotypically and genetically correlated, indicating that mink with high wool density also have thinner guard hair. High genetic and phenotypic correlations were found between guard hair length and thickness. All QTL affecting guard hair length are located at the same positions or in positions with overlapping confidence intervals to QTL affecting guard hair thickness. These findings suggest that the traits to a large extent are under the influence of the same genes or that some of the genes affecting the traits are linked. This finding may have breeding implications, as selection for short guard hair may be simultaneous selection for thin guard hair. In a study of Rambouillet sheep, a SNP associated with staple length and fiber diameter in the rams was found on OAR 10 (Hadfield T.S. \& Cockett N. pers. comm.). This may indicate that it is a biological characteristic shared by other furbearing animals as well. The fact that all the traits with similar QTL positions are genetically correlated suggests that the traits are influenced by some of the same genes (pleiotropic effect) or influenced by genes in very close proximity in linkage disequilibrium. In selection programs, pleiotropic effects prohibit selection for such traits sepa- rately, whereas linkage disequilibrium decays over time due to recombination allowing selection for the traits separately. However, our analysis could not distinguish between these mechanisms.

Some of the alleles originating from the Nordic Brown mink are dominant to alleles originating from short nap mink. Introgressing alleles from American short nap to Nordic Brown may be desirable. However, many of the alleles from American short nap mink with beneficial effects on fur quality are recessive. Conventional selection is not very effective for introgressing recessive alleles. In contrast, marker-assisted selection might be an attractive option. Kenttämies \& Vilva (1988) estimated heritabilities of fur quality traits. However, they found a large variation between color lines and seasons of the year, and the estimates had very large standard errors. Our estimates should therefore be treated with care.

Cadieu et al. (2009) found that very few genes control the variation in guard hair length, coarseness and curly hair in dogs. We found that several QTL affect guard hair length and guard hair thickness. In dogs, it has been shown that short hair is dominant to long hair and that long hair is caused by mutations in the FGF5 gene (e.g., Housley \& Venta 2006). A microsatellite marker (RAN110) developed from the BAC clone containing the FGF5 gene and included in this study did not show any significant association with this trait, despite its very high informativity (Anistoroaei et al. 2012c). However, a non-significant QTL for guard hair length (LOD $=2.58$ ) was found in close proximity to this marker on chromosome 11 in mink.

In dogs, RSPO2 on chromosome 13 affects fur coarseness (Cadieu et al. 2009). The RSPO2 gene is located on mink chromosome 4p (Anistoroaei et al. 2012c). We detected a suggestive QTL affecting guard hair thickness on chromosome $4 \mathrm{p}(\mathrm{LOD}=3.48)$ at position 49 in proximity to marker RAN50.

A low information content observed for some chromosomal areas in this study may bias the results. Potentially, highly significant QTL might be accumulating in the chromosome areas with high information content that follow areas with low information content (Weller 2001). For this reason, multiple QTL affecting different traits might therefore have accumulated at the same position. The analyses of genetic and phenotypic correlations were 
performed based only on phenotypes and were independent of genotypes. As the traits with same QTL position also were genotypically correlated, this may indicate that the traits have similar genetic background and thus support the detected QTL. However, low information content may have reduced our power to detect QTL, and the number of QTL affecting the traits may be underestimated. This might also be the reason for our lack of detecting QTL affecting guard hair length on chromosome 11.

\section{Acknowledgements}

We thank two anonymous reviewers for constructive comments on early drafts of the manuscript. We are very grateful to Per Madsen for technical support on the DMU software and for valuable discussions of results. This work was supported by Janne Thirstrup's Ph.D. scholarship and Razvan Anistoroaei's postdoctoral grant (Danish Research Council \# 27820), as well as funding from Pelsdyrafgiftsfonden and Kopenhagen Fur.

\section{References}

Anistoroaei R. \& Christensen K. (2007) Mapping the silver gene in mink and its association with the dilution gene in dog. Cytogenetic and Genome Research 166, 316-618.

Anistoroaei R., Fredholm M., Kristensen K. \& Leeb T. (2008) Albinism in the American mink (Neovison vison) is associated with a tyrosinase nonsense mutation. Animal Genetics 39, 645-8.

Anistoroaei R., Ansari S., Farid H., Benkel B., Karlskov-Mortensen P. \& Christensen K. (2009) An extended anchored linkage map and virtual mapping for the American mink genome based on homology to human and dog. Genomics 94, 204-10.

Anistoroaei R., ten Hallers B., Nefedov M., Christensen K. \& de Jong P. (2011) Construction of an American mink bacterial artificial chromosome (BAC) library and sequencing candidate genes important for the fur industry. BMC Genomics, 12, 35-4.

Anistoroaei R. Krogh A.K. \& Christensen K. (2012a) A frameshift mutation in the LYST gene is responsible for the Aleutian color and the associated Chédiak-Higashi syndrome in American mink. Animal Genetics. 44, 178-83.

Anistoroaei R., Markakis M.N., Vissenberg K. \& Christensen K. (2012b) Exclusion of candidate genes for coat colour phenotypes of the American mink (Neovison vison). Animal Genetics 43, 813-6.

Anistoroaei R., Nielsen V., Markakis M.N., Karlskov-Mortensen P., Jørgensen C.B., Christensen K. \& Fredholm M. (2012c) A re-assigned American mink (Neovison vison) map optimal for genome-wide studies. Gene 511, 66-72.
Benkel B.F., Rouvinen-Watt K., Farid H. \& Anistoroaei R. (2009) Molecular characterization of the Himalayan mink. Mammalian Genome 20, 256-9.

Cadieu E., Neff M.W., Quignon P. et al. (2009) Coat variation in the domestic dog is governed by variants in three genes. Science $\mathbf{3 2 6}$, 150-3.

Churchill G.A. \& Dorge R.W. (1994) Empirical threshold values for quantitative trait mapping. Genetics 138, 963-71.

Falconer D.S. \& Mackay T.F.C. (1996) Values and means. In: Introduction to Quantitative Genetics, 4th edn, pp. 108-20. Pearson Education Limited.

Haley C.S., Knott S.A. \& Elsen J.-M. (1994) Mapping quantitative trait loci in crosses between outbred lines using least squares. Genetics 136, 1195-207.

Housley D.J.E. \& Venta P.J. (2006) The long and the short of it: evidence that FGF5 is a major determinant of canine 'hair'itability. Animal Genetics 37, 309-15.

Ihaka R. \& Gentleman R. (1996) R: a language for data analysis and graphics. Journal of Computational and Graphical Statistics 5(3), 299-314. R package version 4.5-3, http://CRAN.R-project.org.

Kenttämies H. \& Vilva V. (1988) Phenotypic and genetic parameters for body size and fur characteristics in mink. Acta Agriculturae Scandinavica 38, 243-52.

Madsen P. \& Jensen J. (2010) A User's Guide to DMU. A Package For Analyzing Multivariate Mixed Models. Version 6, release 5.0. University of Aarhus Faculty of Agricultural Science (DJF), http://www.dmu.agrisci.dk/dmu6_guide-R4-6-7.pdf.

Miller S.A., Dykes D.D. \& Polesky H.F. (1988) A simple salting out procedure for extracting DNA from human nucleated cells. Nucleic Acids Research 16, 1215.

Nielsen V.H., Møller S.H., Krogh B. \& Berg P. (2011) Response to selection and genotype-environment interaction in mink (Neovison vison) selected on ad libitum and restricted feeding. Canadian Journal of Animal Science 91, 1-7.

Peakall R. \& Smouse P.E. (2006) Genalex 6: genetic analysis in Excel: population genetic software for teaching and research. Molecular Ecology Notes 6, 288-95.

Seaton G., Haley C.S., Knott S.A., Kearsey M. \& Visscher P.M. (2002) QTL EXPRESS: mapping quantitative trait loci in simple and complex pedigrees. Bioinformatics 18, 339-40.

Visscher P.M., Thompson R. \& Haley C.S. (1996) Confidence intervals for QTL locations using bootstrapping. Genetics 143, 1013-20.

Weller J.I. (2001) Bias in estimation of QTL parameters with interval mapping. In: Quantitative Trait Loci Analysis in Animals, 1st edn, pp. 92. CABI Publishing, UK.

Weller J.I., Kasshi Y. \& Soller M. (1990) Power of daughter and granddaughter designs for determining linkage between marker loci and quantitative trait loci in dairy cattle. Journal of Dairy Science 73, 2525-37. 